\title{
Synthesis and Characterization of Phosphine and Arsine Complexes of Ruthenium (II \& III) Ligated With 3-(4-pyridyl)-4-substituted-triazoline-5-thione
}

\author{
R.N. PANDEY* and NISHA KUMARI \\ P.G. Centre of Chemistry (M.U.), College of Commerce, Patna - 800020 (India) \\ ${ }^{*}$ Corresponding author E-mail: rameshwarnath.pandey@yahoo.com \\ http://dx.doi.org/10.13005/ojc/300480
}

(Received: September 23, 2014; Accepted: November 03, 2014)

\begin{abstract}
Organometallic complexes of ruthenium (II \& III) with the formula $\left[\mathrm{RuH}(\mathrm{CO})\left(\mathrm{E} \phi_{3}\right)_{2} \mathrm{~L}\right]$ and $\left[R u C l_{2}\left(E \phi_{3}\right)_{2} L\right](E=P / A s ; L=$ deprotonated mononegative bidentate 3-(4-pyridyl)-triazoline-5thione and its 4-phenyl substituted derivative) were synthesized and characterized by elemental analysis, physico-chemical and spectroscopic methods. All new compounds were iso-structural with precursor complexes. Two triphenyl phosphine or triphenylarsine molecules are at transdisposition and thioamide ligands behaves as bidentate $(\mathrm{N}, \mathrm{S})$ donor in assigned octahedral structure.
\end{abstract}

Key words: Organo-ruthenium (II \& III), N, S-chelating thioamide, oh-species

\section{INTRODUCTION}

nating ability and interesting insights into structure and bonding ${ }^{1-3}$. Bowman-James's group ${ }^{4-}$ ${ }^{5}$ and Kanbara and Co-worker-8 have reported that thioamide based Pincer complexes are photoluminescent and have catalytic activity. The group of Henderson ${ }^{9-11}$ has reported the synthesis and structural characterization of various transition metal complexes containing chelating thioamide derivatives. The reactions, structures and antitumour activity of transition metal ions with thioamide ligands are reported by Mohr et al ${ }^{12}$. The current interest in organometallic ruthenium complexes with anti-tumour activity ${ }^{13-15}$ prompted us to investigate this class of complexes with 3-(4Pyridyl)-4-substituted-Triazoline-5-thione which contains secondary thioamide group. Prior to this work low-valent $\mathrm{Pd}(0), \mathrm{Pt}(0)$ and $\mathrm{Rh}(\mathrm{I})$ complexes of this ligand are reported in our previous communication ${ }^{16}$. The present study embodies synthesis and characterization of some novel organometallic derivatives of ruthenium (II \& III) with 3-(4-Pyridyl)-4-substituted-triazoline-5-thione (I). 


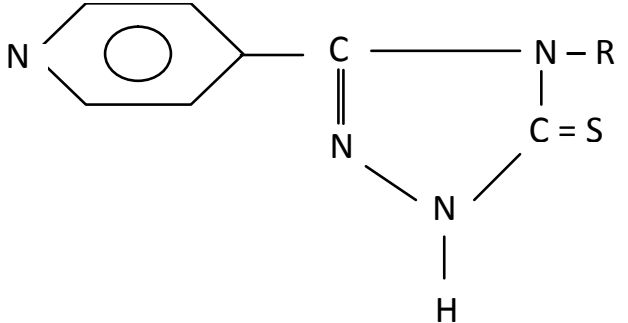

( I )

$$
\left(\mathbf{R}=\mathbf{H} / \mathbf{C}_{6} \mathbf{H}_{5}\right)
$$

\section{EXPERIMENTAL}

All chemicals used were either CP grade or AR grade. Solvents were distilled and dried before use. The ligands 3-(4-Pyridyl)-triazoline-5thione (PytTH) and 3-(4'-Pyridyl)-4-phenyltriazoline-5-thione (PPytTH) were prepared by the method of Dutta et $\mathrm{al}^{17}$. The ligands were recrystallized from ethanol before use. The precursor complexes, $\left[\mathrm{RuX}_{3}\left(\mathrm{Ef}_{3}\right)_{3}\right](\mathrm{X}=\mathrm{Cl} / \mathrm{Br} ; \mathrm{E}=\mathrm{P} /$ $\mathrm{As}),\left[\mathrm{RuH}(\mathrm{CO})\left(\mathrm{Ef}_{3}\right)_{3} \mathrm{Cl}\right](\mathrm{E}=\mathrm{P} / \mathrm{As})$ were prepared following the literature pathway ${ }^{18-22}$.

Preparation of new ruthenium (II) complexes

All ruthenium (II) complexes were prepared by ligand substitution reactions using precursors $\left[\mathrm{RuH}(\mathrm{CO})\left(\mathrm{Ef}_{3}\right)_{3} \mathrm{Cl}\right](\mathrm{E}=\mathrm{P} / \mathrm{As})^{18-19}$ and ethanolic solution of ligands, PytTH or PPytTH in equimolar ratio using our previous method ${ }^{23}$.

\section{Analysis}

S. No.1:

$\left[\mathrm{RuCl}(\mathrm{CO})\left(\mathrm{P} \phi_{3}\right)_{2}(\mathrm{PytT})\right]$

(deep brown) : Calculated (\%) for $\mathrm{RuC}_{44} \mathrm{H}_{35} \mathrm{~N}_{4} \mathrm{OSClP}_{2} \mathrm{Ru}(865.5): \mathrm{C}=$ $61.00 ; \mathrm{H}=5.08 ; \mathrm{N}=6.47 ; \mathrm{Ru}=11.66$; Found $(\%): C=61.18 ; \mathrm{H}=5.18 ; \mathrm{N}=$ 6.50; $\quad \mathrm{Ru}=11.72$;

S. No.2: $\quad\left[\mathrm{RuCl}(\mathrm{CO})\left(\mathrm{As}_{3}\right)_{2}(\mathrm{PytT})\right]$

(Brown) : Calculated (\%) for $\mathrm{RuC}_{44} \mathrm{H}_{35} \mathrm{~N}_{4} \mathrm{OSAs}_{2} \mathrm{Cl}(953.34): \mathrm{C}=$ 55.38; $\mathrm{H}=3.67 ; \mathrm{N}=5.87 ; \mathrm{Ru}=10.59$; Found (\%) : $\mathrm{C}=55.42 ; \mathrm{H}=3.77 ; \mathrm{N}=$ 5.92; $\quad \mathrm{Ru}=10.62$;

S. No.3: $\quad\left[\mathrm{RuCl}(\mathrm{CO})\left(\mathrm{P} \phi_{3}\right)_{2}(\mathrm{PPytT})\right]$ (yellowish brown) : Calculated (\%) for $\mathrm{RuC}_{50} \mathrm{H}_{39} \mathrm{~N}_{4} \mathrm{SP}_{2} \mathrm{Cl}(941.50): \mathrm{C}=63.72$; $\mathrm{H}=4.14 ; \quad \mathrm{N}=5.95 ; \mathrm{Ru}=10.72$;
Found (\%) : $\mathrm{C}=63.82 ; \mathrm{H}=4.21 ; \mathrm{N}=$ 6.02; $\mathrm{Ru}=10.77$;

S. No.4: $\quad\left[\mathrm{RuCl}(\mathrm{CO})\left(\mathrm{As}_{3}\right)_{2}(\mathrm{PPytT})\right]$ (yellowish brown) : Calculated (\%) for $\mathrm{RuC}_{50} \mathrm{H}_{39} \mathrm{~N}_{4}$ $\mathrm{SAs}_{2} \mathrm{Cl}(1025.5): \mathrm{C}=58.50 ; \mathrm{H}=3.80$; $\mathrm{N}=5.46 ; \mathrm{Ru}=9.84 ;$ Found (\%) $: \mathrm{C}=$ 58.62; $\mathrm{H}=3.91 ; \mathrm{N}=5.38 ; \quad \mathrm{Ru}=$ 9.92;

\section{Preparation of ruthenium (III) complexes}

All the reactions were carried out strictly anhydrous conditions and the complexes were prepared using a general method. Benzene solution of $\left[\mathrm{RuX}{ }_{3}\left(\mathrm{E}_{3}\right)_{3}\right](\mathrm{X}=\mathrm{Cl} / \mathrm{Br} ; \mathrm{E}=\mathrm{P} / \mathrm{As})$ and ligand were mixed in equimolar ratios and stirred on magnetic stirrer for 15 mints and heated on reflux till the brown colour changed to yellowish green. The working mixture was concentrated and cooled. Addition of light petroleum ether produced solid products which was filtered and dried in vacuo over anhydrous $\mathrm{CaCl}_{2}$. (yield $\left.=76 \%\right)$

\section{Analysis}

S. No.5: $\quad\left[\mathrm{RuCl}_{2}\left(\mathrm{P}_{3}\right)_{2}(\mathrm{PytT})\right]$ (y ellowish green) : Calculated (\%) for $\mathrm{RuC}_{43} \mathrm{H}_{35} \mathrm{~N}_{4} \mathrm{P}_{2} \mathrm{SCl}_{2}(873): \mathrm{C}=59.10 ; \mathrm{H}$ $=4.00 ; \mathrm{N}=6.41 ; \mathrm{Ru}=11.56 ;$ Found $(\%)$ $: \mathrm{C}=59.01 ; \mathrm{H}=3.98 ; \mathrm{N}=6.38 ; \mathrm{Ru}=$ 11.49;

S. No.6: $\quad\left[\mathrm{RuCl}_{2}\left(\mathrm{As}_{3}\right)_{2}(\mathrm{PytT})\right] \quad$ (g re e n i s h yellow) : Calculated (\%) for $\mathrm{RuC}_{43} \mathrm{H}_{35} \mathrm{~N}_{4}$ $\mathrm{As}_{2} \mathrm{SCl}_{2}(960.84): \mathrm{C}=53.70 ; \mathrm{H}=3.64$; $\mathrm{N}=5.82 ; \mathrm{Ru}=10.51$; Found $(\%): \mathrm{C}=$ 54.01; $\mathrm{H}=3.70 ; \mathrm{N}=5.78 ; \mathrm{Ru}=10.60$;

S. No.7: $\quad\left[\operatorname{RuBr}_{2}\left(\mathrm{P}_{3}\right)_{2}(\mathrm{PytT})\right] \quad$ ( b r o w n is h green) : Calculated (\%) for $\mathrm{RuC}_{43}$ $\mathrm{H}_{35} \mathrm{~N}_{4} \mathrm{P}_{2} \mathrm{SBr}_{2}(962): \mathrm{C}=53.63 ; \mathrm{H}=3.63$; $\mathrm{N}=5.82 ; \mathrm{Ru}=10.49$; Found (\%) $: \mathrm{C}=$ 55.63; $\mathrm{H}=3.68 ; \mathrm{N}=5.80 ; \mathrm{Ru}=10.52$;

S. No.8: $\quad\left[\operatorname{RuBr}_{2}\left(\operatorname{As}_{3}\right)_{2}(\right.$ PytT $\left.)\right] \quad$ ( b r o w n is h green) : Calculated (\%) for $\mathrm{RuC}_{43} \mathrm{H}_{35}$ $\mathrm{N}_{4} \mathrm{As}_{2} \mathrm{SBr}_{2}(1049.74): \mathrm{C}=49.15 ; \mathrm{H}=$ 3.33; $\mathrm{N}=5.33 ; \mathrm{Ru}=9.62 ;$ Found $(\%): \mathrm{C}$ $=49.32 ; \mathrm{H}=3.40 ; \mathrm{N}=5.23 ; \mathrm{Ru}=9.68$;

S. No.9: $\quad\left[\operatorname{RuCl}_{2}\left(\mathrm{P}_{3}\right)_{2}(\mathrm{PPytT})\right]$ (y ellowish green) : Calculated (\%) for $\mathrm{RuC}_{49} \mathrm{H}_{39} \mathrm{~N}_{4}$ $\mathrm{SP}_{2} \mathrm{Cl}_{2}(949): \mathrm{C}=61.95 ; \mathrm{H}=4.10 ; \mathrm{N}=$ 5.90; $\mathrm{Ru}=10.64$; Found (\%) : $\mathrm{C}=62.11$; $\mathrm{H}=4.20 ; \mathrm{N}=6.01 ; \mathrm{Ru}=10.68$;

S. No.10: $\quad\left[\mathrm{RuCl}_{2}\left(\mathrm{Asf}_{3}\right)_{2}(\mathrm{PPytT})\right]$ (yellowish green) 
: Calculated (\%) for $\mathrm{RuC}_{49} \mathrm{H}_{39} \mathrm{~N}_{4}$ $\mathrm{SAs}_{2} \mathrm{Cl}_{2}(1033): \mathrm{C}=56.92 ; \mathrm{H}=4.74 ; \mathrm{N}$ =6.42; $\mathrm{Ru}=9.77$; Found $(\%): \mathrm{C}=$ 57.01; $\mathrm{H}=4.80 ; \mathrm{N}=5.50 ; \mathrm{Ru}=9.89$;

S. No.11: $\left[\operatorname{RuBr}_{2}\left(\mathrm{P}_{\phi_{3}}\right)_{2}(\mathrm{PPytT})\right] \quad$ (g r e e n i s h brown) : Calculated (\%) for $\mathrm{RuC}_{49} \mathrm{H}_{39} \mathrm{~N}_{4}$ $\mathrm{SP}_{2} \mathrm{Br}_{2}(1037.8): \mathrm{C}=56.65 ; \mathrm{H}=3.75$; $\mathrm{N}=5.39 ; \mathrm{Ru}=9.73$; Found $(\%): \mathrm{C}=$ 56.66; $\mathrm{H}=3.88 ; \mathrm{N}=5.42 ; \mathrm{Ru}=9.80$;

S. No.12: $\quad\left[\operatorname{RuBr}_{2}\left(\mathrm{Asf}_{3}\right)_{2}(\mathrm{PPytT})\right] \quad$ (g r e e $\mathrm{n}$ i s h brown) : Calculated (\%) for $\mathrm{RuC}_{49} \mathrm{H}_{39} \mathrm{~N}_{4}$ $\mathrm{SAs}_{2} \mathrm{Br}_{2}(1125.64): \mathrm{C}=52.23 ; \mathrm{H}=3.46$; $\mathrm{N}=4.97 ; \mathrm{Ru}=8.97$; Found $(\%): \mathrm{C}=$ 52.40; $\mathrm{H}=3.50 ; \mathrm{N}=5.01 ; \mathrm{Ru}=9.00$;

The analysis of $\mathrm{C}, \mathrm{H}$ and $\mathrm{N}$ were performed at CDRI, Lucknow, India. IR spectra of ligand and complexes were recorded on a Perkin Elmer 577 spectrophotometer in the range of $4000-200 \mathrm{~cm}^{-1}$ as $\mathrm{KBr}$ pellet technique. Electronic spectra of ligand and complexes were recorded on a Backmann Du6 spectrophotometer. The molar conductance of complexes $\left(10^{-3} \mathrm{M}\right)$ were measured in DMF using Wiss-Werkstatter Weihem obb type LBR conductivity meter. The magnetic susceptibility was measured on a gouy balance using $\mathrm{Hg}\left[\mathrm{CO}(\mathrm{SCN})_{4}\right]$ as calibrant.

\section{RESULTS AND DISCUSSION}

The reactions of $\left[\mathrm{RuHCl}(\mathrm{CO})\left(\mathrm{Ef}_{3}\right)_{2}\right](\mathrm{E}=\mathrm{P} /$ As) (Scheme I) and $\left[\mathrm{RuX}_{3}\left(\mathrm{Ef}_{3}\right)_{3}\right](\mathrm{X}=\mathrm{Cl} / \mathrm{Br} ; \mathrm{E}=\mathrm{P} / \mathrm{As})$ with thioamide ligands (Scheme II) in 1:1 molar ratio in benzene afforded new hexa-coordinated complexes and ligands acts as mononegative bidentate anion.

$$
\begin{array}{r}
{\left[\mathrm{RuHCl}(\mathrm{CO})\left(\mathrm{E}_{3}\right)_{3}\right]+\underset{\substack{\text { or } \\
\text { PPytTH }}}{\frac{\mathrm{C}_{6} \mathrm{H}_{6}}{\text { reflux, } 4-5 \mathrm{hr} .}} \underset{\text { or }}{\left[\mathrm{RuCl}(\mathrm{CO})\left(\mathrm{E} \phi_{3}\right)_{2}(\mathrm{PytT})\right]+\mathrm{E}_{3}+\mathrm{H}_{2}(\mathrm{~g})}} \\
{\left[\mathrm{RuCl}(\mathrm{CO})\left(\mathrm{E} \phi_{3}\right)_{2}(\mathrm{PPytT})\right]}
\end{array}
$$

$$
\begin{array}{r}
\text { Scheme 1: } \\
{\left[\mathrm{RuX}_{3}\left(\mathrm{E}_{3}\right)_{3}\right]+\underset{\text { PytTH }}{\text { or }}} \\
\text { PPytTH }
\end{array}
$$

$$
\begin{array}{r}
\underset{\text { reflux, 4-5 hr. }}{\stackrel{\mathrm{C}_{6} \mathrm{H}_{6}}{\longrightarrow}}\left[\mathrm{RuX}_{2}(\mathrm{E} \phi\right. \\
\mathrm{RuX}_{2}(\mathrm{E}) \\
(\mathrm{X}=\mathrm{Cl} / \mathrm{Br} ; \mathrm{E}=\mathrm{P} / \mathrm{As})
\end{array}
$$

\section{Scheme 2:}

The analytical data are in good agreement with general molecular formula proposed. The mononegative bidentate thioamide ligands replaces are triphenyl phosphine or triphenyl arsine molecule, one chlorido or bromido group from precursors $\left[\mathrm{RuX}_{3}\left(\mathrm{E}_{3}\right)_{3}\right]$ and hydrido group and one $\mathrm{P}_{\phi_{3}}$ or $\mathrm{Asf}_{3}$ from precursors, $\left[\mathrm{RuHCl}(\mathrm{CO})\left(\mathrm{E}_{3}\right)_{3}\right]$. All the isolated products were air-stable and nonhygroscopic nature and fair soluble in acetone, EMK, DMF \& DMSO.

\section{Magnetic moment and UV-Vis Spectra}

The substitution products $[\mathrm{RuCl}(\mathrm{CO})$ $\left.\left(\mathrm{E}_{3}\right)_{2}(\mathrm{PytT})\right]$ and $\left[\mathrm{RuCl}(\mathrm{CO})\left(\mathrm{E}_{3}\right)_{2}(\mathrm{PPytT})\right]$ were diamagnetic indicating $\mathrm{Ru}^{++}\left(\mathrm{d}^{6}\right)$ and ${ }^{1} \mathrm{~A}_{1 \mathrm{~g}}$ ground state. The $d-d$ transition bands (table 2 ) consistent with octahedral structure ${ }^{24}$.

The magnetic moment value of $\left[R u X_{2}\left(E_{3}\right)_{2}(P y t T)\right]$ or $\left[R u X_{2}\left(E_{3}\right)_{2}(P P y t T)\right](X=C l / B r$; $\mathrm{E}=\mathrm{P} / \mathrm{As})$ are in the range of 1.80-1.95 $\mathrm{BM}$ corresponding to a single unpaired electron in a low spin $\mathrm{T}_{2 \mathrm{~g}}{ }_{\text {eg }}{ }^{\circ}$ configuration and oxidation state of ruthenium is +3 . So, the ground state of $R u$ (III) is ${ }^{2} T_{2 g}$ and first excited doublet level in order of increasing energy are ${ }^{2} \mathrm{~A}_{2 g}$ and ${ }^{2} \mathrm{~A}_{19}$ which arises from $\mathrm{t}_{2 \mathrm{~g}}^{4}{ }_{\mathrm{eg}}^{1}$ configuration ${ }^{25}$. The electronic spectra of complexes exhibit bands around $18585\left({ }^{2} \mathrm{~T}_{2 \mathrm{~g}} \rightarrow\right.$ 


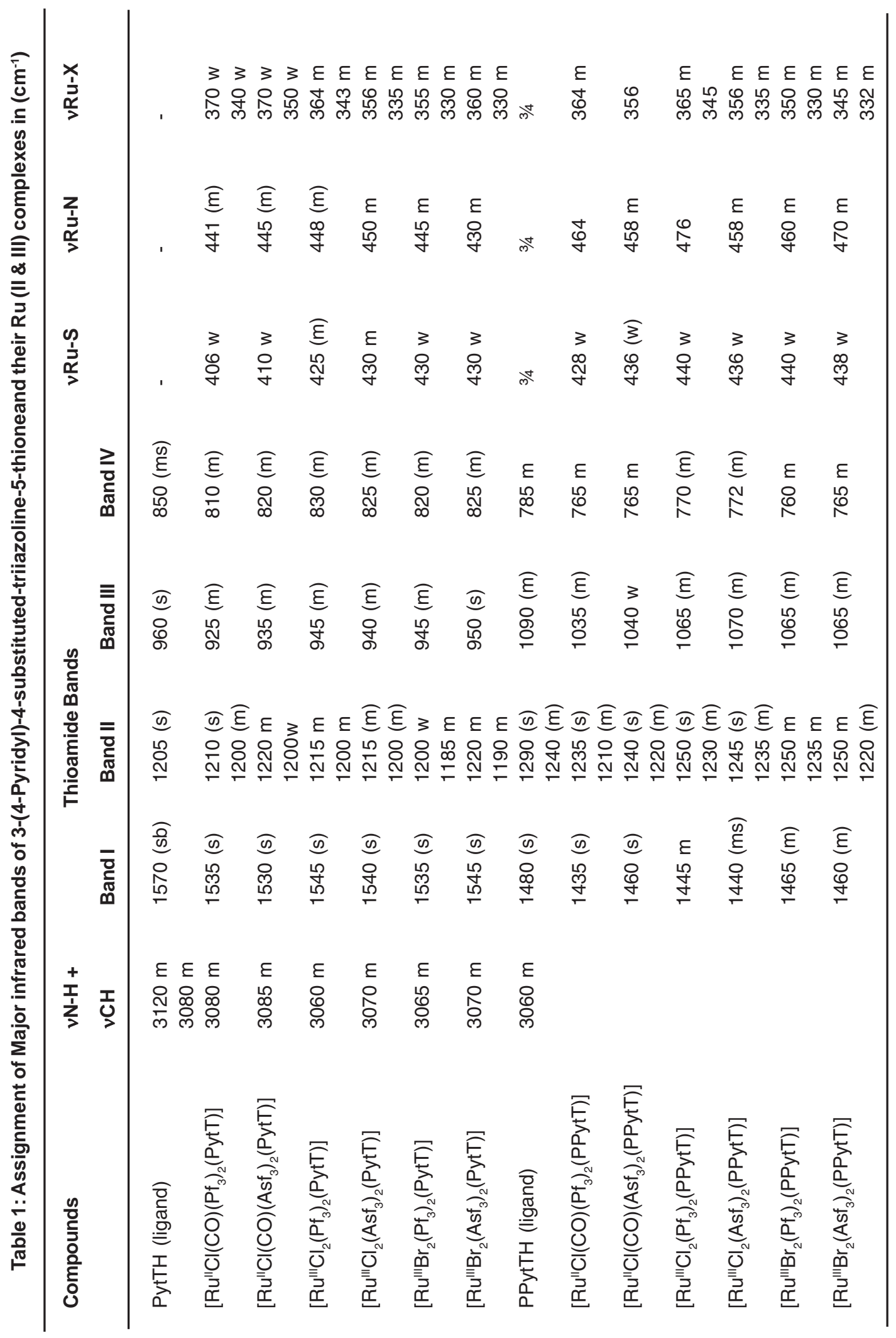


$\left.{ }^{2} A_{2 g}\right), 22935\left(T_{2 g} \rightarrow \pi^{*}, M L C T\right), 38760\left(n \rightarrow \pi^{\star}\right)$ and $49020 \mathrm{~cm}-1\left(\pi \rightarrow \pi^{*}\right)$ are consistent with octahedral structure of other ruthenium (III) complexes ${ }^{26}$.

\section{Infrared Spectra}

The IR bands of interest of ligands and complexe are given in Table 1. A comparison of the infrared bands of the ligands and complexes indicate the simultaneous formation of $\mathrm{Ru}-\mathrm{N}$ and $\mathrm{Ru}-\mathrm{S}$ bonds. (i) Two bands having contribution from $\mathrm{nNH}$ and $\mathrm{nCH}$ are present at $3120 \mathrm{~cm}^{-1}$ and 3080 $\mathrm{cm}^{-1}$ in PytTH and at $3060 \mathrm{~cm}^{-1}$ in PPytTH. But there is only one band centred at 3080 $\mathrm{cm}^{-1}$ in the spectra of complexes of PytTH and found to be absent in the complexes PPytTH. This suggests that $\mathrm{H}$-atom of $\mathrm{N}-\mathrm{H}$ group of ligands has been replaced by the $\mathrm{Ru}(\mathrm{II})$ and $\mathrm{Ru}(\mathrm{III})$ ions leading to the formation of $\mathrm{Ru}^{\prime \prime}-\mathrm{N}$ and $\mathrm{Ru} u^{\prime \prime \prime}-\mathrm{N}$ bonds.

Table 2: Magnetic, Conductometric and Electronic spectral data of ruthenium (II \& III) complexes

\begin{tabular}{|c|c|c|c|}
\hline Complexes & $\mu_{\text {eff. }}(B M)$ & Molar cond. $\Omega^{-1} \mathrm{~cm}^{2} \mathrm{~mol}^{-1}$ & $\lambda_{\max }\left(\mathrm{cm}^{-1}\right)$ (Assignment) \\
\hline$\left[\mathrm{Ru} u^{\prime \prime} \mathrm{Cl}(\mathrm{CO})\left(\mathrm{P}_{\phi_{3}}\right)_{2}(\mathrm{PytT})\right]$ & Dia Mag. & 15.60 & $\begin{array}{c}19240\left({ }^{1} \mathrm{~A}_{1 \mathrm{~g}} \rightarrow{ }^{1} \mathrm{~T}_{1 \mathrm{~g}}\right) \\
21740\left({ }^{1} \mathrm{~A}_{1 \mathrm{~g}}{ }^{1} \mathrm{~T}_{2 \mathrm{~g}}\right) \\
31250(\mathrm{CT} \text { Band })\end{array}$ \\
\hline$\left[\mathrm{Ru} u^{\prime \prime} \mathrm{Cl}(\mathrm{CO})\left(\mathrm{As} \phi_{3}\right)_{2}(\mathrm{PytT})\right]$ & Dia Mag. & 20.60 & $\begin{array}{c}19800\left({ }^{1} \mathrm{~A}_{1 \mathrm{~g}} \rightarrow{ }^{1} \mathrm{~T}_{1 \mathrm{~g}}\right) \\
20405\left({ }^{1} \mathrm{~A}_{1 \mathrm{~g}}{ }^{1} \mathrm{~T}_{2 \mathrm{~g}}\right) \\
28985(\mathrm{CT} \text { Band })\end{array}$ \\
\hline$\left[\mathrm{Ru}^{\mathrm{III}} \mathrm{Cl}_{2}\left(\mathrm{P} \phi_{3}\right)_{2}(\mathrm{PytT})\right]$ & 1.86 & 15.30 & $\begin{array}{c}18585\left({ }^{2} \mathrm{~T}_{2 \mathrm{~g}} \rightarrow{ }^{2} \mathrm{~A}_{2 \mathrm{~g}}\right) \\
22935\left(\mathrm{~T}_{2 \mathrm{~g}} \rightarrow \pi^{*}\right) \\
38760\left(\mathrm{n} \rightarrow \pi^{*}\right) \\
49020\left(\pi \rightarrow \pi^{*}\right)\end{array}$ \\
\hline$\left[\mathrm{Ru}^{\mathrm{III}} \mathrm{Cl}_{2}\left(\mathrm{As} \phi_{3}\right)_{2}(\mathrm{PytT})\right]$ & 1.95 & 15.00 & $\begin{array}{c}18690\left({ }^{2} \mathrm{~T}_{2 \mathrm{~g}} \rightarrow{ }^{2} \mathrm{~A}_{2 \mathrm{~g}}\right) \\
22725\left(\mathrm{~T}_{2 \mathrm{~g}} \rightarrow \pi^{*}\right) \\
37735\left(\mathrm{n} \rightarrow \pi^{\star}\right) \\
48305\left(\pi \rightarrow \pi^{*}\right)\end{array}$ \\
\hline$\left[\mathrm{Ru}^{\prime \prime \prime} \mathrm{Br}_{2}\left(\mathrm{P} \phi_{3}\right)_{2}(\mathrm{PytT})\right]$ & 1.80 & 10.32 & $\begin{array}{c}18690\left({ }^{2} \mathrm{~T}_{2 \mathrm{~g}} \rightarrow{ }^{2} \mathrm{~A}_{2 \mathrm{~g}}\right) \\
22470\left(\mathrm{~T}_{2 \mathrm{~g}} \rightarrow \pi^{*}\right) \\
37735\left(\mathrm{n} \rightarrow \pi^{\star}\right) \\
48780\left(\pi \rightarrow \pi^{*}\right)\end{array}$ \\
\hline$\left[\operatorname{Ru}^{\prime \prime \prime} \mathrm{Br}_{2}\left(\mathrm{As} \phi_{3}\right)_{2}(\mathrm{PytT})\right]$ & 1.90 & 12.30 & $\begin{array}{c}18665\left({ }^{2} \mathrm{~T}_{2 \mathrm{~g}} \rightarrow{ }^{2} \mathrm{~A}_{2 \mathrm{~g}}\right) \\
18510\left(\mathrm{~T}_{2 \mathrm{~g}} \rightarrow \pi^{*}\right) \\
38165\left(\mathrm{n} \rightarrow \pi^{\star}\right) \\
49015\left(\pi \rightarrow \pi^{\star}\right)\end{array}$ \\
\hline$\left[\mathrm{Ru} u^{\prime \prime} \mathrm{Cl}(\mathrm{CO})\left(\mathrm{P}_{\phi_{3}}\right)_{2}(\mathrm{PPytT})\right]$ & Dia, Mag. & 15.35 & $\begin{array}{l}19230\left({ }^{1} \mathrm{~A}_{1 \mathrm{~g}} \rightarrow{ }^{1} \mathrm{~T}_{1 \mathrm{~g}}\right) \\
21740\left({ }^{1} \mathrm{~A}_{1 \mathrm{~g}} \rightarrow{ }^{1} \mathrm{~T}_{2 \mathrm{~g}}\right) \\
34480(\text { CT Band) } \\
29760(\text { CT Band) }\end{array}$ \\
\hline$\left[\mathrm{Ru}^{\prime \prime} \mathrm{Cl}(\mathrm{CO})\left(\mathrm{As} \phi_{3}\right)_{2}(\mathrm{PPytT})\right]$ & Dia, Mag. & 15.30 & $\begin{array}{l}19605\left({ }^{1} \mathrm{~A}_{1 \mathrm{~g}} \rightarrow{ }^{1} \mathrm{~T}_{1 \mathrm{~g}}\right) \\
21740\left({ }^{1} \mathrm{~A}_{1 \mathrm{~g}} \rightarrow{ }^{1} \mathrm{~T}_{2 g}\right) \\
35715(\text { CT Band) } \\
30305 \text { (CT Band) }\end{array}$ \\
\hline 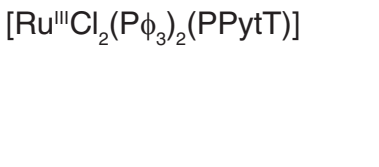 & 1.88 & 10.35 & $\begin{array}{c}18868\left({ }^{2} \mathrm{~T}_{2 \mathrm{~g}} \rightarrow{ }^{2} \mathrm{~A}_{2 \mathrm{~g}}\right) \\
22470\left(\mathrm{~T}_{2 \mathrm{~g}} \rightarrow \mathrm{p}^{*}\right) \\
38460\left(\mathrm{n} \rightarrow \mathrm{p}^{\star}\right) \\
48780\left(\mathrm{p} \rightarrow \mathrm{p}^{\star}\right)\end{array}$ \\
\hline
\end{tabular}




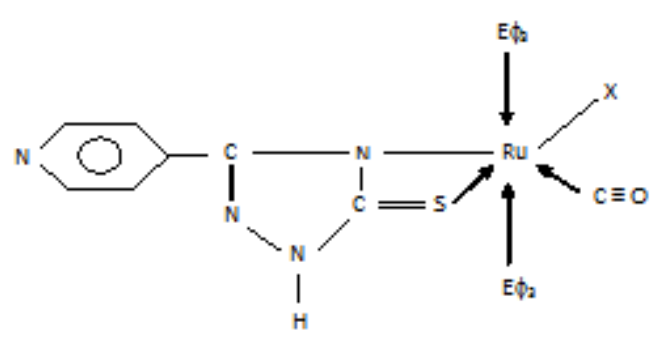

Str. I $(\mathrm{X}=\mathrm{C} / \mathrm{Br} ; \mathrm{E}=\mathrm{P} / \mathrm{As})$

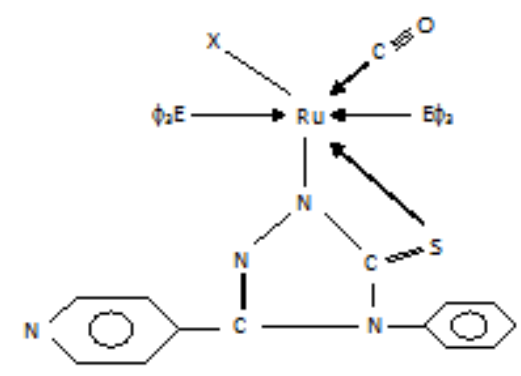

Str. II $(\mathrm{X}=\mathrm{C} / \mathrm{Br} ; \mathrm{E}=\mathrm{P} / \mathrm{As})$

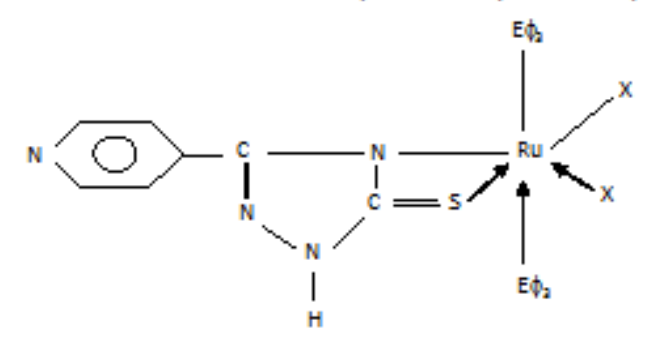

Str. III $(\mathrm{X}=\mathrm{C} / \mathrm{Br} ; \mathrm{E}=\mathrm{P} / \mathrm{As})$ (ii) Thioamide band IV has main contribution from $\mathrm{nC}-\mathrm{S}$ observed at $850 \mathrm{~cm}^{-1}$ (PytTH) and at $785 \mathrm{~cm}^{-1}$ (PPytTH) in the spectra of the ligands shift to lower frequency by $30-50$ $\mathrm{cm}^{-1}$ indicating coordination of thioamide ligands through thione sulphur and formation of ruthenium $-\mathrm{S}$ bond following our previous observations ${ }^{26}$.

(iii) The simultaneous Ru-N and Ru-S bonding results in a decrease in the frequency of thioamide band IV by $30-50 \mathrm{~cm}^{-1}$, blue shift to higher frequency of $10-15 \mathrm{~cm}^{-1}$ of band II and red shift to the extent of $35-55 \mathrm{~cm}^{-1}$ in thioamide band I. These observations are in good agreement with previous literature ${ }^{27}$. New bands at $435-465 \mathrm{~cm}^{-1}$ (Ru-N) and 405$410 \mathrm{~cm}^{-1}$ (Ru-S) due to stretching modes further supports these observations.

(iv) The presence of bands near 540, 680, 750 and $1550 \mathrm{~cm}^{-1}$ are attributed to coordinated $\mathrm{Pf}_{3} / \mathrm{Asf}_{3}$ in complexes ${ }^{29-30}$. The presence of single Ru-P stretching modes at $510-500 \mathrm{~cm}$ ${ }^{1}$ confirms two bulkyu $\mathrm{Pf}_{3}$ groups are at mutual Trans-disposition in octahedral structure of complexes (Str. I \& II). However, more than one Ru-X stretching mode in Ru (III) complexes indicates cis-disposition of two chlorine or two bromine atoms in octahedral structure (Str. III \& IV).

(v) The non-ligand band at $1950-1960 \mathrm{~cm}-1$ in carbonyl complexes of ruthenium (II) supports terminal $\mathrm{C} \cong \mathrm{O}$ group slightly higher frequency than in precursor complex.

Thus, the ligands behaves as mononegative bidentate $(\mathrm{N}, \mathrm{S})$ donor.

\section{REFERENCES}

1. Jagodzinski, T.S., Chem, Rev. 2003, 103, 197.

2. Pandiarajan, D. and Ramesh, R., J. Organometallic Chem. 2013, 723, 26.

3. Murai, T., Top. Curr. Chem. 2005, 251, 247.

4. Begum, R.A., Powell, D., Bowman-James, K., Inorg. Chem. 2006, 45, 964.

5. Hossain, M.A., Lucarini, S., Powell, D. \& Bowman-James, K., Inorg. Chem. 2004, 43, 7275.
6. Kanbara, T., Okada, K., Yamamoto, T., Ogawa, $\mathrm{H}$. and Inoue, T., J. Organomet. Chem. 2004, 689, 1860.

7. Okamoto, K., Kanbara, T., Yamamoto, T. and Wada, T., Organometallics, 2006, 25, 4026.

8. Koizumi, T., Teratani, T., Okamoto, K., Yamamoto, T., Shimoi, Y. and Kanbara, T., Inorg. Chem. Acta, 2010, 363, 2474.

9. Henderson, W., Nicholson, B.K. and Tiekink, E.R.T., Inorg. Chim. Acta, 2006, 359, 204. 
10. Henderson, W. and Rickard, C.E.F., Inorg. Chim. Acta, 2003, 343, 74.

11. Henderson, W., Nicholson, B.K., Dinger, M.B. and Bennett, R.L., Inorg. Chim. Acta, 2002, 338,210

12. Alagoz, C., Brauer, David J. and Mohr Fabian, J. Organometallic Chem. 2009, 694, 1283.

13. Clarke, M.J., Coord. Chem. Rev. 2003, 236, 209.

14. Gielen, M., Tiekink, E.R.T., Metallotherapeutic Drugs and Metal-based Diagnostic Agents, John Wiley and Sons, Chichester, 2005.

15. Yan, Y.K., Melchart, M., Habtemariam, A. and Sadler, P.J., Chem. Commun. 2005, 4764.

16. Pandey, R.N., Kumar Arun, Singh, R.S.P., Sahay, A.N. and Kumar, Shashikant, J. Indian Chem. Soc. 1992, 69, 804.

17. Dutta, S., Acharya, A.K. and Basu, U.P., J. Indian Chem. Soc., 1968, 45, 338.

18. Ahmad, N., Levison, J.J., Robinson, S.D. and Uttley, M.F., Inorg. Synth. 1975, 15, 45.

19. Balasubramanian, K.P., Manivannanand, S. and Chinusamy, V., J. Ultra Chem. 2008, 4,
15.

20. Poddar, R.K., Khullar, I.P. and Agarwala, U., J. Inorg. Nucl. Chem. Lett. 1974, 10, 221.

21. Natarajan, K., Poddar, R.K. and Agarwala, U., J. Inorg. Nucl. Chem. 1977, 39, 431.

22. Chatt, J., Leigh, C.J., Mingos, DMP and Paske, R.J., J. Chem. Soc. A, 1968, 2636.

23. Pandey, R.N., Bala, Renu and Sinha, A.K., Oriental J. Chem. 2011, 27(1), 293.

24. Pandey, R.N. and Gautam, K.V., J. Ultra Chem. 2014, 10(1), 35.

25. Pandiarajan, D. and Ramesh, R., Polyhedron, 2012, 34, 136.

26. Kannan, S. and Ramesh, R., Polyhedron, 2006, 25, 3095.

27. Singh, B., Singh, R., Choudhary, R.V. and Thakur, K.P., Indian J. Chem. 11, 174 (1973).

28. Singh, B. and Singh, R., J. Inorg. Nucl. Chem. 1972, 34, 3449.

29. Pandiarajan, D. and Ramesh, R., J. Organomet. Chem. 2003, 723, 26.

30. Kumar, K.N., Ramesh, R. and Liu, Y., J. Mol. Catal. A Chem., 2007, 265, 218. 